PROCEEDINGS OF THE

AMERICAN MATHEMATICAL SOCIETY

Volume 139, Number 11, November 2011, Pages 3807-3813

S 0002-9939(2011)11083-9

Article electronically published on April 7, 2011

\title{
DEPTH OF EDGE RINGS ARISING FROM FINITE GRAPHS
}

\author{
TAKAYUKI HIBI, AKIHIRO HIGASHITANI, KYOUKO KIMURA, \\ AND AUGUSTINE B. O'KEEFE
}

(Communicated by Irena Peeva)

\begin{abstract}
Let $G$ be a finite graph and $K[G]$ the edge ring of $G$. Based on the technique of Gröbner bases and initial ideals, it will be proved that, given integers $f$ and $d$ with $7 \leq f \leq d$, there exists a finite graph $G$ on $[d]=\{1, \ldots, d\}$ with depth $K[G]=f$ and with Krull-dim $K[G]=d$.
\end{abstract}

\section{INTRODUCTION}

The edge ring [3] and its toric ideal 4 arising from a finite graph have been studied from the viewpoints of both commutative algebra and combinatorics. Especially, the normality of the edge ring as well as the Gröbner bases of its toric ideal is extensively investigated. However, the fundamental question when an edge ring is Cohen-Macaulay is presumably open.

Let $G$ be a finite simple graph, i.e., a finite graph with no loop and no multiple edge, on the vertex set $[d]=\{1, \ldots, d\}$ and $E(G)=\left\{e_{1}, \ldots, e_{r}\right\}$ its edge set. Let $K[\mathbf{t}]=K\left[t_{1}, \ldots, t_{d}\right]$ be the polynomial ring in $d$ variables over a field $K$ and write $K[G]$ for the subring of $K[\mathbf{t}]$ generated by those squarefree quadratic monomials $\mathbf{t}^{e}=t_{i} t_{j}$ with $e=\{i, j\} \in E(G)$. The semigroup ring $K[G]$ is called the edge ring of $G$. Let Krull-dim $K[G]$ denote the Krull dimension of $K[G]$ and depth $K[G]$ the depth of $K[G]$. Let $K[\mathbf{x}]=K\left[x_{1}, \ldots, x_{r}\right]$ be the polynomial ring in $r$ variables over a field $K$. The kernel $I_{G}$ of the surjective homomorphism $\pi: K[\mathbf{x}] \rightarrow K[G]$ defined by setting $\pi\left(x_{i}\right)=\mathbf{t}^{e_{i}}$ for $i=1, \ldots, r$ is called the toric ideal of $G$. One has $K[G] \cong K[\mathbf{x}] / I_{G}$. If $G$ is connected and is nonbipartite (resp. bipartite), then Krull-dim $K[G]=d$ (resp. Krull-dim $K[G]=d-1$ ).

The criterion of normality [3, Corollary 2.3] of edge rings guarantees that $K[G]$ is normal if either $G$ is bipartite or $d \leq 6$. If $d=7$, then there exists a finite graph $G$ for which $K[G]$ is nonnormal. However, it follows easily that $K[G]$ is CohenMacaulay whenever $d \leq 7$. Computing the depth of the edge rings of all connected nonbipartite graphs $G$ with 7 vertices shows that the depth of $K[G]$ is at least 7 . Moreover, our computational experiment would naturally lead the authors into the temptation to give the following conjecture.

Received by the editors September 8, 2010.

2010 Mathematics Subject Classification. Primary 13P10.

Key words and phrases. Edge ring, toric ideal, finite graph, Gröbner basis, initial ideal.

The fourth author had summer support provided by the JSPS Research Fellowships for Young Scientists and the NSF East Asia and Pacific Institutes Fellowship.

(C)2011 American Mathematical Society Reverts to public domain 28 years from publication 
Conjecture 0.1. Let $G$ be a finite connected nonbipartite graph on $[d]$ with $d \geq 7$. Then $\operatorname{depth} K[G] \geq 7$.

Now, even though Conjecture 0.1 is completely open, by taking Conjecture 0.1 into consideration, this paper will be devoted to proving the following.

Theorem 0.2. Given integers $f$ and $d$ with $7 \leq f \leq d$, there exists a finite graph $G$ on $[d]$ with $\operatorname{depth} K[G]=f$ and with Krull-dim $K[G]=d$.

Let $k \geq 1$ be an arbitrary integer and $G_{k+6}$ the finite graph on $[k+6]$ of Figure 0.1. The essential part of a proof of Theorem 0.2 is to show that

$$
\operatorname{depth} K\left[G_{k+6}\right]=\operatorname{depth} K[\mathbf{x}] / I_{G_{k+6}}=7 \text {. }
$$

In Section 1, by virtue of the formula [1, Theorem 2.1], the inequality depth $K\left[G_{k+6}\right]$ $\leq 7$ will be proved. In Section 2, we compute a Gröbner basis of $I_{G_{k+6}}$ and an initial ideal in $\left(I_{G_{k+6}}\right)$ of $I_{G_{k+6}}$, and show the inequality depth $K[\mathbf{x}] / \operatorname{in}\left(I_{G_{k+6}}\right) \geq 7$. In general, one has depth $K[\mathbf{x}] / I_{G_{k+6}} \geq \operatorname{depth} K[\mathbf{x}] / \operatorname{in}\left(I_{G_{k+6}}\right)$ (e.g., [2, Theorem 3.3.4 (d)]). Thus the desired equality (0.1) follows.

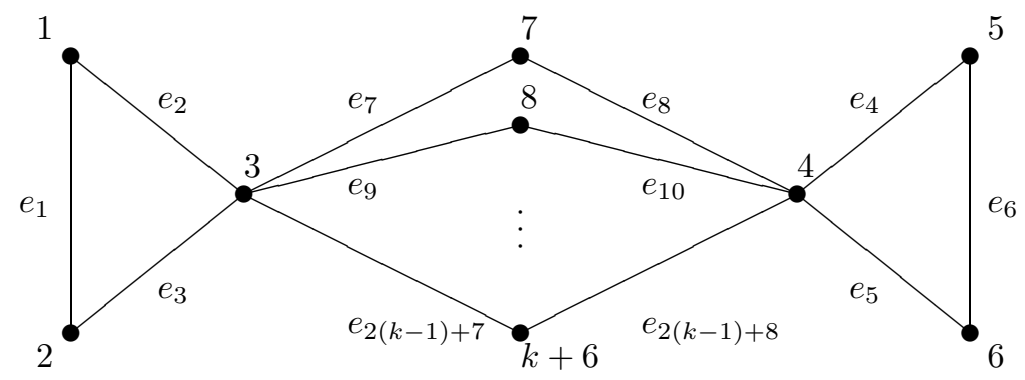

FIGURE 0.1. (finite graph $G_{k+6}$ )

Once we know that depth $K\left[G_{k+6}\right]=7$, to prove Theorem 0.2 is straightforward. In fact, given integers $f$ and $d$ with $7 \leq f \leq d$, let $\Gamma$ denote the finite graph $G_{d-f+7}$ on $[d-f+7]$ and write $G$ for the finite graph on $[d]$ obtained from $\Gamma$ by adding $f-7$ edges

$$
\{1, d-f+8\},\{1, d-f+9\}, \ldots,\{1, d\}
$$

to $\Gamma$. It then follows that $\operatorname{depth} K[G]=\operatorname{depth} K[\Gamma]+f-7$. Since depth $K[\Gamma]=7$, one has $\operatorname{depth} K[G]=f$, as required.

\section{Proof of depth $K\left[G_{k+6}\right] \leq 7$}

Let $G=G_{k+6}$ of Figure 0.1. In this section, we prove that depth $K[G] \leq 7$. Since the number of edges of $G$ is $r=2(k-1)+8$, the Auslander-Buchsbaum formula implies that we may prove pd $K[G] \geq r-7=2 k-1$.

Let $S_{G}$ be the semigroup arising from $G$. Let $\mathcal{A}_{G}=\left\{\underline{a}_{1}, \ldots, \underline{a}_{r}\right\}$ be the set of columns of the incidence matrix of $G$ where $\underline{a}_{l}$ corresponds to the edge $e_{l}$ (which corresponds to the variable $x_{l}$ ). Therefore, $S_{G}=\mathbb{N} \mathcal{A}_{G}$.

To prove pd $K[G] \geq 2 k-1$, we use the following theorem due to Briales, Campillo, Marijuán, and Pisón [1]. For $\underline{s} \in S_{G}$, we define the simplicial complex

$$
\Delta_{\underline{s}}=\left\{F \subset[r]: \underline{s}-\underline{n}_{F} \in S_{G}\right\},
$$


where $\underline{n}_{F}=\sum_{l \in F} \underline{a}_{l}$. We denote by $\beta_{i, \underline{s}}(K[G])$, the $i$ th multigraded Betti number of $K[G]$ in degree $\underline{s}$.

Lemma 1.1 ([1, Theorem 2.1]). Let $G$ be a finite simple graph. Then

$$
\beta_{j+1, \underline{s}}(K[G])=\operatorname{dim}_{K} \tilde{H}_{j}\left(\Delta_{\underline{s}} ; K\right) .
$$

We consider the case where

$$
\underline{s}=(1,1, k+1, k+1,1,1,2,2, \ldots, 2) .
$$

By Lemma 1.1, it is sufficient to prove the following lemma:

Lemma 1.2. Set $\underline{s}=(1,1, k+1, k+1,1,1,2,2, \ldots, 2)$. Then

$$
\operatorname{dim}_{K} \tilde{H}_{2 k-2}\left(\Delta_{\underline{s}} ; K\right) \neq 0 .
$$
$\Delta$.

We set $\Delta=\Delta_{s}$. Before proving Lemma 1.2, we compute the simplicial complex

Lemma 1.3. Set $\underline{s}=(1,1, k+1, k+1,1,1,2,2, \ldots, 2)$. Then the facets of $\Delta_{\underline{s}}$ are the following subsets of $[r]$ :

$$
\begin{array}{ll}
F_{1, i}=\{1,4,5,7,8, \ldots, 2(k-1)+8\} \backslash\{2(i-1)+8\}, & i=1, \ldots, k ; \\
F_{2, j}=\{2,3,6,7,8, \ldots, 2(k-1)+8\} \backslash\{2(j-1)+7\}, & j=1, \ldots, k .
\end{array}
$$

Proof. Since $\underline{s}-\underline{n}_{F_{1, i}}=\underline{a}_{2(i-1)+7} \in S_{G}$, we have $F_{1, i} \in \Delta_{\underline{s}}=\Delta$. (It follows that $\underline{s} \in S_{G}$.) Similarly, we have $F_{2, j} \in \Delta$.

To prove that there are no facets other than $F_{1, i}, F_{2, j}$, it is enough to show that

- $\{1,2\},\{1,3\},\{4,6\},\{5,6\} \notin \Delta$;

- $\{1,6\} \notin \Delta$

- $\{2,4\},\{2,5\},\{3,4\},\{3,5\} \notin \Delta$

- $F_{0}=\{7,8, \ldots, 2(k-1)+8\} \notin \Delta$.

Since the first entry of $\underline{s}-\underline{n}_{\{1,2\}}$ is $-1<0$, it follows that $\underline{s}-\underline{n}_{\{1,2\}} \notin S_{G}$. Therefore $\{1,2\} \notin \Delta$. By symmetry, we also have $\{1,3\},\{4,6\},\{5,6\} \notin \Delta$.

Second we show that $\{1,6\} \notin \Delta$. Suppose, on the contrary, that $\{1,6\} \in \Delta$, i.e.,

$$
\underline{s}-\underline{n}_{\{1,6\}}=(0,0, k+1, k+1,0,0,2,2, \ldots, 2) \in S_{G} .
$$

Then we can write $\underline{s}-\underline{n}_{\{1,6\}}=\sum_{l=1}^{r} c_{l} \underline{a}_{l}$, where $c_{l} \in \mathbb{N}$. Since $\left(\underline{s}-\underline{n}_{\{1,6\}}\right)_{1}=$ $\left(\underline{s}-\underline{n}_{\{1,6\}}\right)_{2}=0$ and $\left(\underline{s}-\underline{n}_{\{1,6\}}\right)_{3}=k+1$, we have $c_{1}=c_{2}=c_{3}=0$ and $\sum_{i=1}^{k} c_{2(i-1)+7}=k+1$. Similarly, we have $c_{4}=c_{5}=c_{6}=0$ and $\sum_{j=1}^{k} c_{2(j-1)+8}=$ $k+1$. Then $\sum_{i=1}^{k} c_{2(i-1)+7}+\sum_{j=1}^{k} c_{2(j-1)+8}=2(k+1)$, but it must be $2 k$. This is a contradiction.

Next we show that $\{2,4\},\{2,5\},\{3,4\},\{3,5\} \notin \Delta$. Suppose that $\{2,4\} \in \Delta$, i.e.,

$$
\underline{s}-\underline{n}_{\{2,4\}}=(0,1, k, k, 0,1,2,2, \ldots, 2) \in S_{G} .
$$

Then we can write $\underline{s}-\underline{n}_{\{2,4\}}=\sum_{l=1}^{r} c_{l} \underline{a}_{l}$, where $c_{l} \in \mathbb{N}$. Since $\left(\underline{s}-\underline{n}_{\{2,4\}}\right)_{1}=0$ and $\left(\underline{s}-\underline{n}_{\{2,4\}}\right)_{2}=1$, we have $c_{3}=1$. Similarly, we have $c_{5}=1$. Thus

$$
(0,0, k-1, k-1,0,0,2,2, \ldots, 2) \in S_{G} .
$$

Then a similar argument to the proof of $\{1,6\} \notin \Delta$ yields a contradiction. Therefore $\{2,4\} \notin \Delta$. By symmetry, we also have $\{2,5\},\{3,4\},\{3,5\} \notin \Delta$.

Last, we show $F_{0} \notin \Delta$. It follows from

$$
\underline{s}-\underline{n}_{F_{0}}=(1,1,1,1,1,1,0,0, \ldots, 0) \notin S_{G} .
$$


Now we prove Lemma 1.2 .

Proof of Lemma 1.2. Let $\Delta_{1}$ be the subcomplex of $\Delta$ whose facets are $F_{1, i}, i=$ $1, \ldots, k$, and let $\Delta_{2}$ be the subcomplex of $\Delta$ whose facets are $F_{2, j}, j=1, \ldots, k$. Then $\Delta=\Delta_{1} \cup \Delta_{2}$. Also, the facets of the simplicial complex $\Delta_{1} \cap \Delta_{2}$ are

$$
\{7,8, \ldots, 2(k-1)+8\} \backslash\{2(j-1)+7,2(i-1)+8\}, \quad i, j=1, \ldots, k .
$$

In particular, $\operatorname{dim}\left(\Delta_{1} \cap \Delta_{2}\right)=2 k-3$. Note that both of $\Delta_{1}$ and $\Delta_{2}$ are cones over some simplicial complexes, and so the reduced homologies of all of these vanish. Therefore the Mayer-Vietoris sequence

$$
\begin{aligned}
\cdots & \longrightarrow \tilde{H}_{i}\left(\Delta_{1} \cap \Delta_{2} ; K\right) \longrightarrow \tilde{H}_{i}\left(\Delta_{1} ; K\right) \oplus \tilde{H}_{i}\left(\Delta_{2} ; K\right) \longrightarrow \tilde{H}_{i}(\Delta ; K) \\
& \longrightarrow \tilde{H}_{i-1}\left(\Delta_{1} \cap \Delta_{2} ; K\right) \longrightarrow \tilde{H}_{i-1}\left(\Delta_{1} ; K\right) \oplus \tilde{H}_{i-1}\left(\Delta_{2} ; K\right) \longrightarrow \cdots
\end{aligned}
$$

yields

$$
\tilde{H}_{i}(\Delta ; K) \cong \tilde{H}_{i-1}\left(\Delta_{1} \cap \Delta_{2} ; K\right) \quad \text { for all } i .
$$

We can see that $\tilde{H}_{2 k-3}\left(\Delta_{1} \cap \Delta_{2} ; K\right) \neq 0$ by considering the alternating sum of all facets of $\Delta_{1} \cap \Delta_{2}$ :

$$
\sum_{1 \leq i, j \leq k}(-1)^{i+j}\{7,8, \ldots, 2(k-1)+8\} \backslash\{2(j-1)+7,2(i-1)+8\} .
$$

Therefore we have $\tilde{H}_{2 k-2}(\Delta ; K) \neq 0$.

\section{Proof of depth $K\left[G_{k+6}\right] \geq 7$}

As before, let $G=G_{k+6}$ be as in Figure 0.1. In this section we prove that depth $K[G] \geq 7$.

We set $C_{1}=\left(e_{2}, e_{1}, e_{3}\right)$ and $C_{2}=\left(e_{4}, e_{6}, e_{5}\right)$, both of which are 3-cycles of $G$. By [4, Lemma 3.2], there are 3 kinds of primitive even closed walks $\Gamma$ of $G$ up to route:

(I) a 4-cycle: $\Gamma=\left(e_{2(i-1)+7}, e_{2(i-1)+8}, e_{2(j-1)+8}, e_{2(j-1)+7}\right)$, where $i<j$;

(II) a walk on two 3 -cycles $C_{1}, C_{2}$ and a single path connecting $C_{1}$ and $C_{2}$ : $\Gamma=\left(C_{1}, e_{2(i-1)+7}, e_{2(i-1)+8}, C_{2}, e_{2(i-1)+8}, e_{2(i-1)+7}\right)$, where $i=1, \ldots, k$;

(III) a walk on two 3 -cycles $C_{1}, C_{2}$ and two different paths combining $C_{1}$ and $C_{2}: \Gamma=\left(C_{1}, e_{2(i-1)+7}, e_{2(i-1)+8}, C_{2}, e_{2(j-1)+8}, e_{2(j-1)+7}\right)$, where $i<j$.

It was proved in [4, Lemma 3.1] that binomials corresponding to these primitive even closed walks generate the toric ideal $I_{G}$. Let us consider the lexicographic order $<=<_{\text {lex }}$ with $x_{1}>x_{2}>x_{3}>\cdots>x_{2(k-1)+8}$.

Lemma 2.1. The set of binomials corresponding to primitive even closed walks (I), (II), (III) is a Gröbner basis of $I_{G}$ with respect to $<_{\text {lex }}$.

Proof. The result follows from a straightforward application of Buchberger's algorithm to the set of generators of $I_{G}$ corresponding to the primitive even closed walks listed above. Let $f$ and $g$ be two such generators. We will prove that the $S$-polynomial, $S(f, g)$, arising from Buchberger's algorithm will reduce to 0 by generators of type (I), (II) and (III). For convenience of notation, we will assume that $i, j, p$, and $q$ are all odd integers such that $7 \leq i<j, 7 \leq p<q$. 
Case 1. Let $f=x_{i} x_{j+1}-x_{i+1} x_{j}$ and $g=x_{p} x_{q+1}-x_{p+1} x_{q}$ be generators of type (I). If $i \neq p$ and $j \neq q$, then the leading terms of $f$ and $g$ are relatively prime and thus the $S$-polynomial $S(f, g)$ will reduce to 0 (e.g., [2, Lemma 2.3.1]). Suppose $i=p$; then

$$
\begin{aligned}
S(f, g) & =\frac{\operatorname{cm}\left(L M_{<_{\text {lex }}}(f), L M_{<_{\text {lex }}}(g)\right)}{L T_{<_{\text {lex }}}(f)} f-\frac{\operatorname{lcm}\left(L M_{<_{\text {lex }}}(f), L M_{<_{\text {lex }}}(g)\right)}{L T_{<_{\text {lex }}}(g)} g \\
& =x_{q+1}\left(x_{i} x_{j+1}-x_{i+1} x_{j}\right)-x_{j+1}\left(x_{i} x_{q+1}-x_{i+1} x_{q}\right) \\
& =x_{i+1} x_{j+1} x_{q}-x_{i+1} x_{j} x_{q+1} \\
& =x_{i+1}\left(x_{j+1} x_{q}-x_{j} x_{q+1}\right) .
\end{aligned}
$$

Note that, up to sign, $x_{j+1} x_{q}-x_{j} x_{q+1}$ is a generator of $I_{G}$ of type (I) and therefore $S(f, g)$ will reduce to 0 . The case of $j=q$ is similar.

Case 2. Let $f$ be the same as above and $g=x_{1} x_{4} x_{5} x_{p}^{2}-x_{2} x_{3} x_{6} x_{p+1}^{2}$ be a generator of type (II). If $i \neq p$, then the leading terms of $f$ and $g$ are relatively prime and therefore negligible. If $i=p$, then

$$
\begin{aligned}
S(f, g) & =x_{1} x_{4} x_{5} x_{i}\left(x_{i} x_{j+1}-x_{i+1} x_{j}\right)-x_{j+1}\left(x_{1} x_{4} x_{5} x_{i}^{2}-x_{2} x_{3} x_{6} x_{i+1}^{2}\right) \\
& =x_{2} x_{3} x_{6} x_{i+1}^{2} x_{j+1}-x_{1} x_{4} x_{5} x_{i} x_{i+1} x_{j} \\
& =-x_{i+1}\left(x_{1} x_{4} x_{5} x_{i} x_{j}-x_{2} x_{3} x_{6} x_{i+1} x_{j+1}\right),
\end{aligned}
$$

where $x_{1} x_{4} x_{5} x_{i} x_{j}-x_{2} x_{3} x_{6} x_{i+1} x_{j+1}$ is a generator of type (III).

Case 3. Again, we assume that $f$ is the same as above. Now assume $g$ is of type (III), $g=x_{1} x_{4} x_{5} x_{p} x_{q}-x_{2} x_{3} x_{6} x_{p+1} x_{q+1}$. If $i \neq p, q$, then the leading terms of $f$ and $g$ will be relatively prime. Suppose $i=p$; then

$$
\begin{aligned}
S(f, g) & =x_{1} x_{4} x_{5} x_{q}\left(x_{i} x_{j+1}-x_{i+1} x_{j}\right)-x_{j+1}\left(x_{1} x_{4} x_{5} x_{i} x_{q}-x_{2} x_{3} x_{6} x_{i+1} x_{q+1}\right) \\
& =-x_{i+1}\left(x_{1} x_{4} x_{5} x_{q} x_{j}-x_{2} x_{3} x_{6} x_{q+1} x_{j+1}\right)
\end{aligned}
$$

and again we have that $x_{1} x_{4} x_{5} x_{q} x_{j}-x_{2} x_{3} x_{6} x_{q+1} x_{j+1}$ is either a type (II) or type (III) generator of $I_{G}$. The case of $i=q$ is similar.

Case 4. Now let $f$ and $g$ both be generators of type (II), $f=x_{1} x_{4} x_{5} x_{i}^{2}-x_{2} x_{3} x_{6} x_{i+1}^{2}$, $g=x_{1} x_{4} x_{5} x_{j}^{2}-x_{2} x_{3} x_{6} x_{j+1}^{2}$. Then the $S$-polynomial

$$
\begin{aligned}
S(f, g) & =x_{j}^{2}\left(x_{1} x_{4} x_{5} x_{i}^{2}-x_{2} x_{3} x_{6} x_{i+1}^{2}\right)-x_{i}^{2}\left(x_{1} x_{4} x_{5} x_{j}^{2}-x_{2} x_{3} x_{6} x_{j+1}^{2}\right) \\
& =x_{2} x_{3} x_{6}\left(x_{i}^{2} x_{j+1}^{2}-x_{i+1}^{2} x_{j}^{2}\right) \\
& =x_{2} x_{3} x_{6}\left(x_{i} x_{j+1}+x_{i+1} x_{j}\right)\left(x_{i} x_{j+1}-x_{i+1} x_{j}\right)
\end{aligned}
$$

is a multiple of a type (I) generator.

Case 5. Let $f$ be the same as in Case 4 and $g=x_{1} x_{4} x_{5} x_{p} x_{q}-x_{2} x_{3} x_{6} x_{p+1} x_{q+1}$ be of type (III). First suppose that $i \neq p, q$. Let us consider the case of $i<p$. Then

$$
\begin{aligned}
S(f, g) & =x_{p} x_{q}\left(x_{1} x_{4} x_{5} x_{i}^{2}-x_{2} x_{3} x_{6} x_{i+1}^{2}\right)-x_{i}^{2}\left(x_{1} x_{4} x_{5} x_{p} x_{q}-x_{2} x_{3} x_{6} x_{p+1} x_{q+1}\right) \\
& =x_{2} x_{3} x_{6}\left(x_{i}^{2} x_{p+1} x_{q+1}-x_{i+1}^{2} x_{p} x_{q}\right) \\
& =x_{2} x_{3} x_{6}\left[x_{i} x_{q+1}\left(x_{i} x_{p+1}-x_{i+1} x_{p}\right)+x_{i} x_{i+1} x_{p} x_{q+1}-x_{i+1}^{2} x_{p} x_{q}\right] \\
& =x_{2} x_{3} x_{6}\left[x_{i} x_{q+1}\left(x_{i} x_{p+1}-x_{i+1} x_{p}\right)+x_{i+1} x_{p}\left(x_{i} x_{q+1}-x_{i+1} x_{q}\right)\right],
\end{aligned}
$$

and so $S(f, g)$ reduces to 0 by two type (I) generators. The cases of $p<i<q$ and $q<i$ are similar. 
Now suppose $i=p$. Then the $S$-polynomial,

$$
\begin{aligned}
S(f, g) & =x_{q}\left(x_{1} x_{4} x_{5} x_{i}^{2}-x_{2} x_{3} x_{6} x_{i+1}^{2}\right)-x_{i}\left(x_{1} x_{4} x_{5} x_{i} x_{q}-x_{2} x_{3} x_{6} x_{i+1} x_{q+1}\right) \\
& =x_{2} x_{3} x_{6} x_{i+1}\left(x_{i} x_{q+1}-x_{i+1} x_{q}\right),
\end{aligned}
$$

is a multiple of a type (I) generator. The case of $i=q$ is similar.

Case 6. Finally, we consider the case that both $f$ and $g$ are of type (III): $f=$ $x_{1} x_{4} x_{5} x_{i} x_{j}-x_{2} x_{3} x_{6} x_{i+1} x_{j+1}, g=x_{1} x_{4} x_{5} x_{p} x_{q}-x_{2} x_{3} x_{6} x_{p+1} x_{q+1}$. We may assume that $i \leq p$. Let us first suppose that $i, j \neq p, q$. Then

$$
\begin{aligned}
S(f, g)= & x_{p} x_{q}\left(x_{1} x_{4} x_{5} x_{i} x_{j}-x_{2} x_{3} x_{6} x_{i+1} x_{j+1}\right) \\
& -x_{i} x_{j}\left(x_{1} x_{4} x_{5} x_{p} x_{q}-x_{2} x_{3} x_{6} x_{p+1} x_{q+1}\right) \\
= & x_{2} x_{3} x_{6}\left(x_{i} x_{j} x_{p+1} x_{q+1}-x_{i+1} x_{j+1} x_{p} x_{q}\right) \\
= & x_{2} x_{3} x_{6}\left[x_{j} x_{q+1}\left(x_{i} x_{p+1}-x_{i+1} x_{p}\right)+x_{i+1} x_{p}\left(x_{j} x_{q+1}-x_{j+1} x_{q}\right)\right] .
\end{aligned}
$$

Now let $i=p$. We then have

$$
\begin{aligned}
S(f, g) & =x_{q} f-x_{j} g=-x_{q} x_{2} x_{3} x_{6} x_{i+1} x_{j+1}+x_{j} x_{2} x_{3} x_{6} x_{i+1} x_{q+1} \\
& =x_{2} x_{3} x_{6} x_{i+1}\left(x_{j} x_{q+1}-x_{j+1} x_{q}\right) .
\end{aligned}
$$

The cases of $j=p$ and $j=q$ are similar.

Now we prove that depth $K[G] \geq 7$. We denote by $\operatorname{in}\left(I_{G}\right)$ the initial ideal of $I_{G}$ with respect to $<_{\text {lex }}$. Since

$$
\operatorname{depth} K[G]=\operatorname{depth} K[\mathbf{x}] / I_{G} \geq \operatorname{depth} K[\mathbf{x}] / \operatorname{in}\left(I_{G}\right),
$$

it is sufficient to prove that depth $K[\mathbf{x}] / \operatorname{in}\left(I_{G}\right) \geq 7$. By the Auslander-Buchsbaum formula, it is enough to prove the following lemma:

\section{Lemma 2.2.}

$$
\operatorname{pd}_{K[\mathbf{x}]} K[\mathbf{x}] / \operatorname{in}\left(I_{G}\right) \leq 2 k-1 .
$$

Proof. First we compute in $\left(I_{G}\right)$.

The binomials corresponding to type (I) are

$$
x_{2(i-1)+7} x_{2(j-1)+8}-x_{2(i-1)+8} x_{2(j-1)+7}, \quad \text { where } i<j .
$$

The initial term of this binomial is $x_{2(i-1)+7} x_{2(j-1)+8}(i<j)$. We denote by $I^{\prime}$ the ideal generated by these monomials. Note that $x_{8}$ and $x_{2(k-1)+7}$ do not appear in the minimal system of monomial generators of $I^{\prime}$.

The binomials corresponding to types (II), (III) are

$$
x_{2} x_{3} x_{6} x_{2(i-1)+8} x_{2(j-1)+8}-x_{1} x_{4} x_{5} x_{2(i-1)+7} x_{2(j-1)+7}, \quad \text { where } i \leq j .
$$

The initial term of this binomial is $-x_{1} x_{4} x_{5} x_{2(i-1)+7} x_{2(j-1)+7}(i \leq j)$.

Therefore

$$
\begin{aligned}
\operatorname{in}\left(I_{G}\right) & =x_{1} x_{4} x_{5}\left(x_{7}, x_{9}, \ldots, x_{2(k-1)+7}\right)^{2}+I^{\prime} \\
& =\left(\left(x_{7}, x_{9}, \ldots, x_{2(k-1)+7}\right)^{2}+I^{\prime}\right) \cap\left(\left(x_{1} x_{4} x_{5}\right)+I^{\prime}\right) .
\end{aligned}
$$

We set

$$
\begin{aligned}
& I_{1}=\left(x_{7}, x_{9}, \ldots, x_{2(k-1)+7}\right)^{2}+I^{\prime}, \\
& I_{2}=\left(x_{1} x_{4} x_{5}\right)+I^{\prime} .
\end{aligned}
$$


By the short exact sequence

$$
0 \rightarrow K[\mathbf{x}] / I_{1} \cap I_{2} \rightarrow K[\mathbf{x}] / I_{1} \oplus K[\mathbf{x}] / I_{2} \rightarrow K[\mathbf{x}] /\left(I_{1}+I_{2}\right) \rightarrow 0,
$$

we have

$\operatorname{pd}_{K[\mathbf{x}]} K[\mathbf{x}] / \operatorname{in}\left(I_{G}\right) \leq \max \left\{\operatorname{pd}_{K[\mathbf{x}]} K[\mathbf{x}] / I_{1}, \operatorname{pd}_{K[\mathbf{x}]} K[\mathbf{x}] / I_{2}, \operatorname{pd}_{K[\mathbf{x}]} K[\mathbf{x}] /\left(I_{1}+I_{2}\right)-1\right\}$.

Now we investigate each of $\operatorname{pd}_{K[\mathbf{x}]} K[\mathbf{x}] / I_{1}, \operatorname{pd}_{K[\mathbf{x}]} K[\mathbf{x}] / I_{2}, \operatorname{pd}_{K[\mathbf{x}]} K[\mathbf{x}] /\left(I_{1}+I_{2}\right)$. First we consider the ideal $I_{1}$. Note that $x_{1}, \ldots, x_{6}$ and $x_{8}$ do not appear in the minimal system of monomial generators of $I_{1}$. Let $K\left[\mathbf{x}^{\prime}\right]$ be the polynomial ring over $K$ with variables $x_{7}, x_{9}, x_{10}, \ldots, x_{2(k-1)+8}$. Then $\operatorname{pd}_{K[\mathbf{x}]} K[\mathbf{x}] / I_{1}=\operatorname{pd}_{K\left[\mathbf{x}^{\prime}\right]} K\left[\mathbf{x}^{\prime}\right] /\left(I_{1} \cap\right.$ $\left.K\left[\mathbf{x}^{\prime}\right]\right)$. By Hilbert's syzygy theorem, we have $\operatorname{pd}_{K\left[\mathbf{x}^{\prime}\right]} K\left[\mathbf{x}^{\prime}\right] /\left(I_{1} \cap K\left[\mathbf{x}^{\prime}\right]\right) \leq 2 k-1$.

Next we consider the ideal $I_{2}=\left(x_{1} x_{4} x_{5}\right)+I^{\prime}$. Since the variables $x_{1}, x_{4}, x_{5}$ do not appear in the minimal system of generators of $I^{\prime}$, we have

$$
\operatorname{pd}_{K[\mathbf{x}]} K[\mathbf{x}] / I_{2}=\operatorname{pd}_{K[\mathbf{x}]} K[\mathbf{x}] / I^{\prime}+\operatorname{pd}_{K[\mathbf{x}]} K[\mathbf{x}] /\left(x_{1} x_{4} x_{5}\right)=\operatorname{pd}_{K[\mathbf{x}]} K[\mathbf{x}] / I^{\prime}+1 .
$$

Then similarly to the case of $I_{1}$, we have $\operatorname{pd}_{K[\mathbf{x}]} K[\mathbf{x}] / I^{\prime} \leq 2 k-2$. Thus we have $\operatorname{pd}_{K[\mathbf{x}]} K[\mathbf{x}] / I_{2} \leq 2 k-1$.

Last, we consider the ideal $I_{1}+I_{2}=\left(x_{1} x_{4} x_{5}\right)+I_{1}$. For the same reason as the case of $I_{2}$, we have $\operatorname{pd}_{K[\mathbf{x}]} K[\mathbf{x}] /\left(I_{1}+I_{2}\right)=\operatorname{pd}_{K[\mathbf{x}]} K[\mathbf{x}] / I_{1}+1 \leq 2 k$.

Combining these results with (2.1), we have $\operatorname{pd}_{K[\mathbf{x}]} K[\mathbf{x}] / \operatorname{in}\left(I_{G}\right) \leq 2 k-1$, as desired.

\section{REFERENCES}

[1] E. Briales, A. Campillo, C. Marijuán, and P. Pisón, Combinatorics of syzygies for semigroup algebras, Collect. Math. 49 (1998), 239-256. MR1677160 (99m:13024)

[2] J. Herzog and T. Hibi, "Monomial Ideals", Graduate Texts in Math., 260, Springer, 2011.

[3] H. Ohsugi and T. Hibi, Normal polytopes arising from finite graphs, J. Algebra 207 (1998), 409-426. MR 1644250 (2000a:13010)

[4] H. Ohsugi and T. Hibi, Toric ideals generated by quadratic binomials, J. Algebra 218 (1999), 509-527. MR 1705794(2000f:13055)

Department of Pure and Applied Mathematics, Graduate School of Information Science and Technology, Osaka University, Toyonaka, Osaka 560-0043, Japan

E-mail address: hibi@math.sci.osaka-u.ac.jp

Department of Pure and Applied Mathematics, Graduate School of Information Science and Technology, Osaka University, Toyonaka, Osaka 560-0043, Japan

E-mail address: sm5037ha@ecs.cmc.osaka-u.ac.jp

Department of Mathematics, Faculty of Science, Shizuoka University, 836 Ohya, Suruga-Ku, SHIZUOKA 422-8529, JAPAN

E-mail address: skkimur@ipc.shizuoka.ac.jp

Department of Mathematics, Tulane University, 6823 St. Charles Avenue, New OrLEANS, LoUisiana 70118

E-mail address: aokeefe@tulane.edu 